\title{
PANORAMA DA SUSTENTABILIDADE EM GESTÃO DE PROJETOS
}

Lorena Gamboa Abadia (lorenagabadia@gmail.com) - Departamento de Engenharia de Produção, Escola Politécnica, Universidade de São Paulo.

Marly Monteiro de Carvalho (marlymc@usp.br) - Departamento de Engenharia de Produção, Escola Politécnica, Universidade de São Paulo.

\section{RESUMO}

A relação entre gerenciamento de projetos e desenvolvimento sustentável vem ganhando o interesse de profissionais e acadêmicos ao longo dos últimos anos. Sabe-se que a sustentabilidade é um tema emergente dentro e fora da gestão de projetos, sendo essencial para o bom posicionamento e o consequente sucesso de um projeto, que sua gestão considere aspectos da sustentabilidade. Este trabalho foi elaborado com o objetivo de analisar a evolução do uso da sustentabilidade na literatura científica de gestão de projetos dos últimos dez anos, de forma a identificar o desenvolvimento $e$ relevância do tema para a academia, analisando os principais temas nessa interface. Ao mesmo tempo, apresentando um panorama que possa vir a motivar futuras pesquisas. A abordagem metodológica da pesquisa contempla técnicas de revisão da literatura e análise de conteúdo. Os principais resultados apontam para a multidisciplinaridade do tema sustentabilidade, abordado por inúmeras áreas, ainda dentro do meio da gestão de projetos, que é em si um tópico que envolve também diversas áreas. Nota-se que existe um interesse crescente sobre a sustentabilidade no âmbito da gestão de projetos. As principais publicações na interconexão dos dois assuntos tratam da inclusão da sustentabilidade à ciência da gestão de projetos, da gestão de projetos sustentáveis ou questões da área da construção civil.

Palavras-chave: gestão de projetos; sustentabilidade; revisão da literatura.

Área: Gestão de projetos de desenvolvimento de produtos e serviços.

\section{INTRODUÇÃO}

Nas últimas décadas, a questão ambiental tem ganhado cada vez mais espaço em debates, normas e padrões internacionais, o que reflete uma mudança na legislação interna de muitos países e no posicionamento de organizações. As atividades humanas se adaptaram a hábitos mais sustentáveis, a partir de lições aprendidas com o ecossistema natural (YANG; ZOU, 2014). Estados, empresas e diversos setores da sociedade percebem a gravidade dos impactos causados pelas atividades humanas sobre o meio ambiente, e a necessidade de se promoverem mudanças no sentido de reduzir a degradação ambiental.

A definição de sustentabilidade apresentada por Elkington (1998) considera as dimensões sociais, ambientais e econômicas como pilares, o tripé conhecido como Tripple Bottom Line. Assim, Carvalho e Rabechini Jr. (2011) argumentam a necessidade de essas dimensões serem inseridas e trabalhadas no ofício da gestão de projetos, em direção à sustentabilidade empresarial. Dessa forma, a gestão de projetos pode ser uma maneira de influenciar positivamente a integração de dimensões de sustentabilidade em projetos (BOCCHINI et al., 2014). 
Os principais guias de referência na área de gerenciamento de projetos, oferecidos por organizações importantes como Project Management Institute (PMI, 2013), International Project Management Association (IPMA, 2013), Australian Institute of Project Management (AIPM, 2013) e Association for Project Management (APM, 2013), não dedicam atenção especial à questão da sustentabilidade, como já destacado por Martens e Carvalho (2016). No entanto, a relação entre gerenciamento de projetos e desenvolvimento sustentável está ganhando rapidamente interesse de profissionais e acadêmicos (SILVIUS; SCHIPPERB; NEDESKI, 2013).

Dessa forma, o presente trabalho foi elaborado com o objetivo de analisar a condição da interação entre Gestão de Projetos e Sustentabilidade no meio acadêmico e profissional, através de uma revisão das publicações científicas dos últimos dez anos, de forma a identificar o desenvolvimento e relevância de ambos os temas para a academia, analisando os principais tópicos e abordagens na interface dessas temáticas, e apresentando um panorama que possa vir a motivar futuras pesquisas. As amostras utilizadas na elaboração do estudo foram retiradas da base do ISI, Web of Science.

\section{REVISÃO DA LITERATURA}

Atualmente, compreende-se na sociedade a necessidade da busca por um desenvolvimento sustentável, que pode ser entendido como um desenvolvimento que comporte atender as necessidades das atuais gerações, permitindo ainda que as futuras gerações também possam atender suas necessidades (WCED, 1987). Assim, sabe-se também que diversos fatores tem impacto no desenvolvimento sustentável, incluindo o consumo acelerado de recursos, a geração de resíduos, poluição, e o aumento da população, pobreza e desigualdades sociais (HART; MILSTEIN, 2003).

Diversos autores tem estudado os aspectos da inclusão da sustentabilidade pelas organizações. Porter e Kramer (2011) discutem a criação de valor compartilhado, promovendo soluções capazes de criar valor econômico e, ao mesmo tempo, atender às necessidades da sociedade. Os desafios da sustentabilidade podem ser vistos como oportunidades de inovação empresarial para abordar as incertezas associadas, investigar novas soluções e envolver os recursos necessários para implementar esses desenvolvimentos (YORK; VENKATARAMAN, 2010). Há, assim, uma demanda por um modelo de gestão de negócios que faça a conexão entre a criação de valor e a compatibilidade ecológica e social, unindo essas ideias em equilíbrio (MARTENS; CARVALHO, 2016; AL-SALEH; TALEB, 2010; VEREIN DEUTSCHER INGENIEURE, 2006).

\subsection{Sustentabilidade em gestão de projetos}

Os aspectos de desenvolvimento sustentável apresentam-se ainda de forma incipiente no âmbito da gestão de projetos (CARVALHO; RABECHINI JR., 2011), porém, é necessária uma participação cada vez maior. Seja no meio acadêmico ou no prático, a atenção para a sustentabilidade é crescente e pode ser considerada uma tendência emergente (SILVIUS; SCHIPPERB; NEDESKI, 2013). Pode-se dizer que é essencial para o bom posicionamento de um projeto e, consequentemente, seu sucesso, que a sua gestão considere aspectos da sustentabilidade, desde questões sociais, como saúde e segurança (CARVALHO; RABECHINI JR., 2011), até questões relacionadas ao impacto das atividades sobre o meio ambiente ao longo de todo o ciclo de vida do projeto.

Projetos que buscam seguir pilares sustentáveis, agregando valor a aspectos sociais e ambientais, requerem adaptações às praticas tradicionais, de forma a minimizar riscos e 
melhorar as condições de custos (ROBICHAUD; ANANTATMULA, 2011). Além disso, com a crescente priorização de questões como a sustentabilidade, a proteção do ambiente e as alterações climáticas, o papel do gerente de projeto deve ser adaptado (HWANG; NG, 2013). $\mathrm{O}$ aumento do interesse e da atenção à sustentabilidade em projetos é conduzido pelos valores pessoais dos gerentes de projetos, dos patrocinadores e da estratégia da empresa (SILVIUS; SCHIPPERB; NEDESKI, 2013).

Assim, pode-se afirmar que esse é um meio que se encontra em processo de mudança. Empresas tem se organizado para responder de maneira mais eficaz e ágil a questões ambientais, com foco especialmente na competição e no posicionamento de mercado (CARVALHO; RABECHINI JR., 2011).

\section{MÉTODO}

A abordagem metodológica desta pesquisa consiste de uma revisão da literatura suportada por análise de conteúdo. O processo de pesquisa e análise de conteúdo qualitativo adaptado de Mayring (2014) consistiu em três etapas: coleta de materiais, seleção de categorias e avaliação de materiais. Carvalho, Lopes e Marzagão (2013) recomendam a análise de conteúdo para entendimento aprofundado do tema, possibilitando identificar os principais obstáculos e tendências. O objetivo desse processo é delinear as principais linhas de pesquisa, bem como desencadear novas investigações (SEURING; MÜLLER, 2008).

Para seleção da amostra, realizou-se uma busca na base de dados Web of Science, reconhecida por sua relevância no meio acadêmico. A busca foi realizada utilizando-se os tópicos "project management" AND "sustainab*", de forma a incluir apenas publicações relacionadas simultaneamente aos dois temas. O símbolo (*) é utilizado com a função de incluir na amostra variações ortográficas de termos relacionados a sustentabilidade (como sustainable ou sustainability).

Em seguida, as publicações foram filtradas segundo ano e tipo de publicação, sendo incluídos artigos e reviews, dos anos 2007 a 2016. Esse processo resultou em 184 publicações, sobre as quais se realizou a leitura de títulos, palavras-chave e resumos. A partir dessa leitura, foi possível selecionar as publicações a serem mantidas ou excluídas da amostra. De acordo com o direcionamento do estudo, mantiveram-se as publicações que tratavam de gestão de projetos e sustentabilidade, considerando-se a definição do tripé proposto por Elkington (1998). Foram excluídas 29 publicações que não se adequavam a essas condições, ou por não tratar de gestão de projetos ou por não abordar as três vertentes da sustentabilidade. A amostra final contém 155 artigos, os quais foram separados em 10 categorias, de acordo com o principal tema abordado.

\section{RESULTADOS}

Os resultados desta pesquisa buscam analisar a evolução do uso da sustentabilidade na literatura científica de gestão de projetos dos últimos dez anos, de forma a identificar o desenvolvimento e relevância do tema para a academia, analisando os principais assuntos e abordagens nessa interface. A partir da análise dos resultados, é possível perceber que o número de publicações sobre o tema é crescente dentro do período estudado. Observa-se que os estudos na área continuam em desenvolvimento, o que demonstra esse interesse crescente sobre a sustentabilidade em gestão de projetos.

\subsection{Fontes de publicação}


Foram selecionadas, então, 155 publicações, que se originam de 99 diferentes fontes, sendo na maioria periódicos das áreas de gestão, engenharia, construção civil, ciências ambientais, economia, ciência sociais e de desenvolvimento urbano ou rural, o que possibilita uma visão prévia sobre as principais áreas temáticas na intersecção da gestão de projetos e sustentabilidade. Na figura 1 podem-se observar as seis principais fontes das publicações incluídas na amostra, sendo a primeira delas o Journal of Cleaner Production, com 11 publicações, seguido do Jounal of Management in Engineering com 8, o Sustainability e o International Journal of Project Management, ambos com 6 publicações, Proceedings of the Institution of Civil Engineering - Engineering Sustainability com 5 e o Project Management Journal com 4 publicações. Além dos journals mencionados, a amostra apresenta ainda outras 4 fontes com 3 publicações, 14 com 2 publicações, e 76 fontes com apenas 1 publicação, que não foram incluídas no gráfico.

Figura 1 - principais fontes de publicação da amostra

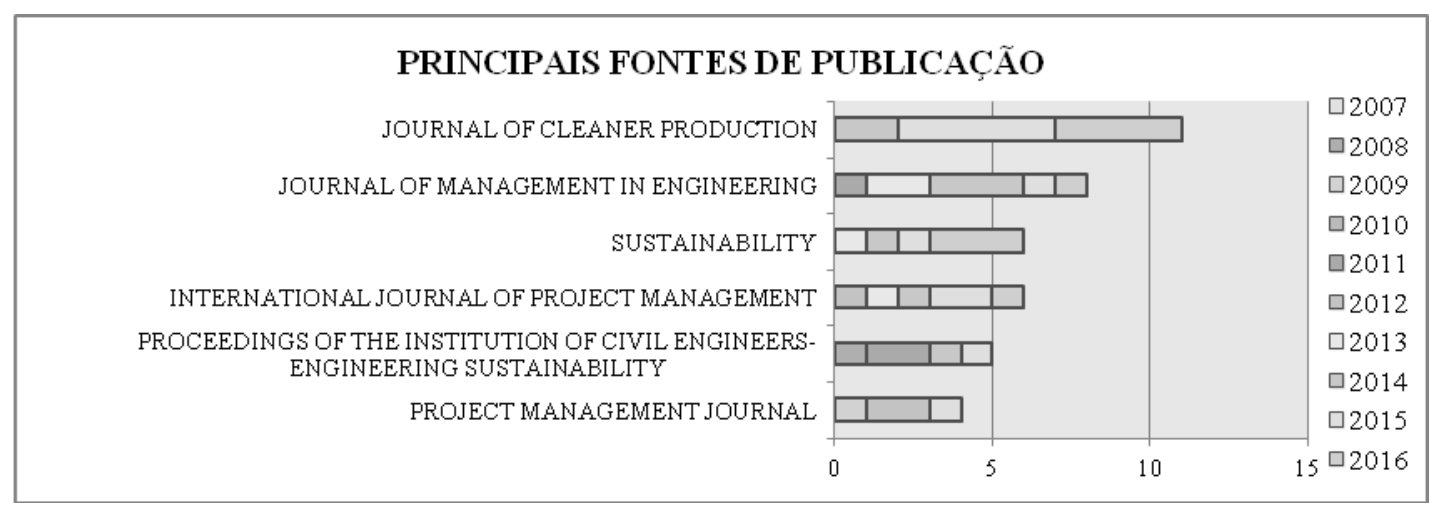

\subsection{Temas das publicações}

A partir da leitura de títulos, palavras-chave e resumos, os artigos da amostra foram categorizados. Os clusters (Figura 2) foram definidos de acordo com os temas abordados pelos artigos, agrupados a partir da leitura. Ainda que diversos artigos se relacionem a mais de um tema ou cluster, uma vez que estes se entrelaçam, o que diferencia a seleção do cluster (quando há sobreposição) é o foco principal do estudo. Por exemplo, projetos de construções de rodovias - alguns tratam sobre o ponto de vista da mobilidade, infraestrutura e logística e outros tratam do ponto de vista da construção civil e do projeto de engenharia em si. Nesses casos, o ponto de vista utilizado no artigo como foco principal foi o que definiu o cluster escolhido.

O primeiro tema é o de Gestão de Projetos Sustentáveis (GPS). Nessa categoria incluem-se os trabalhos que abordam a gestão de projetos como ciência, a inclusão da sustentabilidade como vantagem competitiva em projetos e organizações (MARCELINO-SADABA; GONZALEZJAEN; PEREZ-EZCURDIA, 2015; SANCHEZ, 2015), inclusão do conhecimento de comunidade locais (local expertise) ou tradicionais para a gestão de projetos (GÖRG et al., 2014) ou a gestão de projetos sustentáveis (GUERRERO-LIQUET et al., 2016) como sugere o nome da categoria. 
Figura 2- classificações da amostra

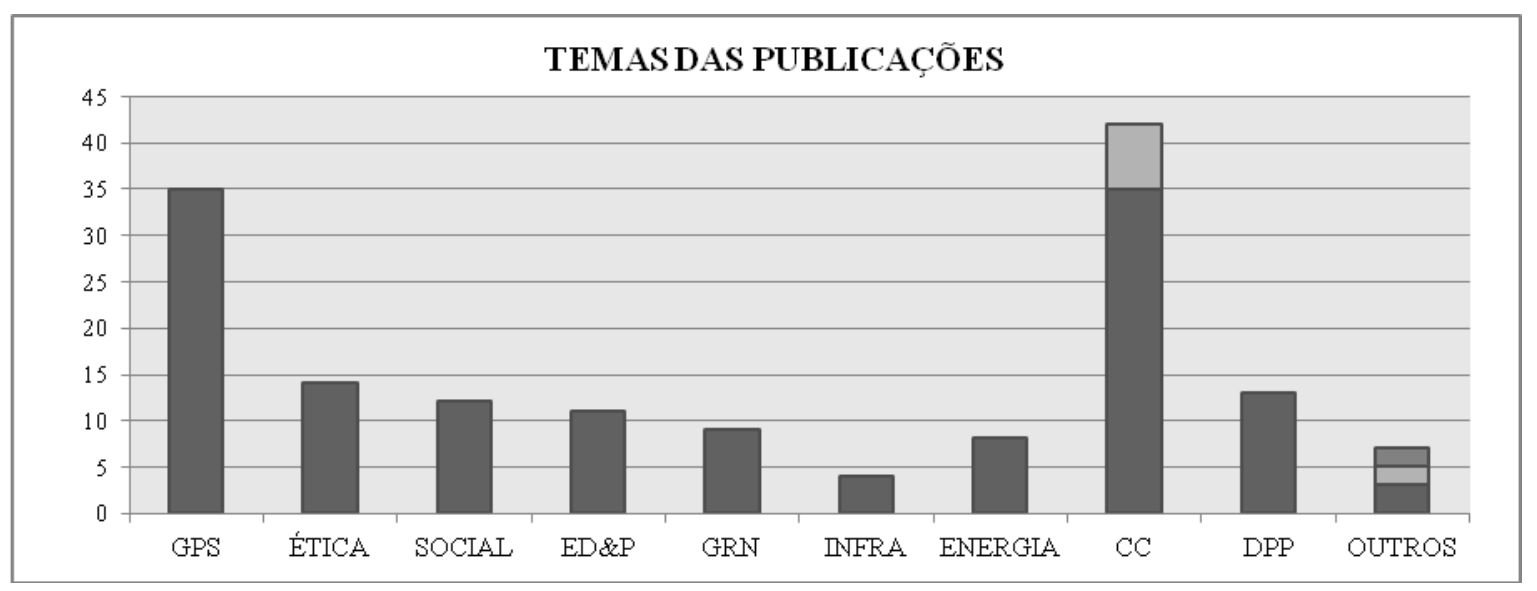

O segundo grupo, nomeado ÉTICA, inclui publicações que tratam de assuntos relacionados a ética em projetos e organizações, compliance (LAWS; LOEBER, 2011), gestão e relacionamento com stakeholders (YANG; ZOU, 2014; YANG; SHEN, 2015) governança (GHOSH, 2014), políticas e legislação (CUST, 2009).

O cluster SOCIAL agrupa as publicações que abordam a sustentabilidade especialmente no âmbito das questões sociais como desenvolvimento de comunidades urbanas (ALMANSI; TAMMARAZIO, 2008) ou rurais (CAZORLA; DE LOS RIOS; SALVO, 2013), aspectos de saúde e saneamento básico (OKEIBUNOR, 2012), e projetos com ONGs. ED\&P refere-se a Educação e Pesquisa, e agrupa publicações como, por exemplo, Trencher et al (2014) que trata de parcerias entre universidades para a concepção e produção de sustentabilidade urbana.

O cluster GRN refere-se a Gestão de Recursos Naturais. Mais variado, este grupo inclui publicações de pesquisa sobre atividades de impacto ambiental, como mineração (CRAIG; SAYDAM; DEMPSTER, 2014), produção de combustíveis (MADDEN; MORAWSKI, 2011), e piscicultura (ZHANG et al., 2009). Além de estudos relacionados à gestão de recursos hídricos (ISON; ROLING; WATSON, 2011), e à gestão de recursos naturais em um sentido mais ligado a ecologia e preservação, gestão de áreas de preservação, recuperação de áreas degradadas (COLENBRANDER; SOWMAN, 2015).

O grupo INFRA, com apenas quatro publicações, é o menor cluster, e agrupa os trabalhos que abordam questões de infraestrutura, logística e urbanismo (LEHEIS, 2012; MIEG, 2012; SENEVIRATNE; AMARATUNGA; HAIGH, 2015; DE BRUIJNE et al, 2010). Como sugere o nome, o cluster ENERGIA inclui os estudos relacionados a geração e gestão de energia, por exemplo, em busca de formas mais sustentáveis de geração (BALEZENTIENE; STREIMIKIENE; BALEZENTIS, 2013) ou do uso mais eficiente (AFLAKI; KLEINDORFER; POLVORINOS, 2013).

A coluna $\mathrm{CC}$ é a que apresenta o maior número de publicações e refere-se à área de construção civil. Nota-se um subgrupo dentro dessa coluna, com sete publicações, que inclui os estudos que tratam especificamente de construções sustentáveis, os chamados Green Buildings (ARASZKIEWICZ , 2016; HERAZO, B; LIZARRALDE, 2015; HWANG; NG, 2013; HWANG; TAN, 2012; MOLENAAR.; SOBIN.; ANTILLON, 2010; ROBICHAUD; ANANTATMULA, 2011; WEERASINGHE; SOUNDARARAJAN; RUWANPURA, 2007).

Depois, temos o cluster de estudos sobre Desenvolvimento de Produtos e Processos, com 13 publicações, incluindo principalmente estudos relacionados a ecodesign (BONOU; SKELTON; OLSEN, 2016; BRONES; DE CARVALHO, 2015; BRONES; DE 
CARVALHO; ZANCUL, 2014, LUIZ et al., 2016) e a gestão ou análise do ciclo de vida (GMELIN; SEURING, 2014; JIAO et al., 2013; SANDIN et al., 2014).

Na última coluna foram agrupados os estudos relacionados a Tecnologias da Informação primeiro subgrupo (GHAPANCHI, 2015; PADE-KHENE; MALLINSON; SEWRY, 2011); Parcerias Público-Privadas - segundo subgrupo (DE LOS RIOS-CARMENADO; ORTUNO; RIVERA, 2016; KAKABADSE; KAKABADSE; SUMMERS, 2007). E, também, artigos que, segundo a opinião das autoras, não se enquadraram nas categorias definidas, como turismo e rotulagem ambiental (LEBE; VRECKO, 2015), avaliação de padrões (PETTER; KHAZANCHI; MURPHY, 2010) e ergonomia (VIDAL et al., 2012) - terceiro subgrupo.

\section{DISCUSSÃO}

Ao observar as categorias definidas a partir da leitura dos artigos, é possível notar que duas delas se destacam das demais, sendo elas a de Gestão de Projetos Sustentáveis - o que era de se esperar dadas as características de busca e seleção da amostra - e a de Construção Civil. Sobre a primeira, no momento da análise de conteúdo foi possível perceber que muitos artigos utilizam-se do termo sustentável referindo-se apenas o aspecto financeiro. Ou outras vezes incluem a diretriz ambiental, mas ainda deixam de fora a diretriz social. Ou seja, muitos dos trabalhos que, pela leitura do resumo e palavras-chaves, pareciam tratar de sustentabilidade, na verdade referiam-se ao termo sustentável considerando apenas o âmbito financeiro. $\mathrm{O}$ que mostra que ainda há muito espaço para a produção de pesquisas que realmente considerem o tripé da sustentabilidade na gestão de projetos como ciência e sua inclusão como vantagem competitiva em projetos e organizações.

Os estudos da área de construção civil também se destacam na amostra. Atualmente, a introdução do desenvolvimento sustentável tanto no planejamento urbano como nos projetos de construção é um fato reconhecido (FERNÁNDEZ-SÁNCHEZ; RODRÍGUEZ-LÓPEZ, 2010). Projetos de construção são ambientes dinâmicos que resultam em circunstâncias de alta incerteza e riscos (TAYLAN et al., 2014), e também são projetos que apresentam bastante impacto sobre o meio ambiente (YANG; ZOU, 2014) de forma que os riscos de sustentabilidade ambiental não devem ser ignorados em tais projetos.

É também devido a essas características próprias dos projetos de construção civil que o interesse nos chamados green buildings é cada vez maior, tanto na academia quanto na prática. As características únicas das construções sustentáveis exigem ajustes nas práticas tradicionais de gerenciamento de projetos para minimizar riscos e melhorar as condições para custos aceitáveis (ROBICHAUD, ANANTATMULA; 2011). Um gerente de projetos precisa de conhecimentos e competências críticos para executar de maneira eficaz um projeto de construção verde (HWANG; NG, 2013).

Assim, dentre os artigos da amostra, uma grande parte trata da área de construção civil, sendo que alguns abordam uma visão de gestão de projetos, através da seleção de projetos de construção e avaliação de risco (TAYLAN et al., 2014), identificação de indicadores de sustentabilidade na gestão de projetos de construção (FERNÁNDEZ-SÁNCHEZ; RODRÍGUEZ-LÓPEZ, 2010), e sugestão de modificações específicas às práticas de construção convencionais para projetos de construção sustentável (ROBICHAUD; ANANTATMULA, 2011). Enquanto que outros abordam questões específicas do gerente de projetos, como conhecimentos e competências dos gestores de projetos no contexto da construção verde (HWANG; NG, 2013), ou um sistema de apoio à decisão multicritérios para avaliação de gestores de projetos em construção (ZAVADSKAS et al., 2012). 


\section{CONCLUSÕES}

O presente estudo se propôs a realizar uma revisão da literatura com análise de conteúdo, com o objetivo de analisar a evolução do uso da sustentabilidade na literatura científica de gestão de projetos dos últimos dez anos. Os resultados apresentados apontam que o tópico da sustentabilidade é um tema de interesse crescente no âmbito acadêmico e prático da gestão de projetos.

A partir das 155 publicações da amostra, foram encontradas publicações sobre diversos temas, o que mostra que a sustentabilidade é um assunto abordado por diversas áreas, ainda dentro do meio da gestão de projetos, que é em si um tópico que envolve também inúmeras áreas. Porém destaca-se um grande interesse nessa interface por parte da área de construção civil, o que é justificado pelas características próprias da área, uma vez que essa apresenta bastante impacto sobre o meio ambiente. Além disso, outros temas observados na amostra foram relacionados diretamente a inclusão da vertente de sustentabilidade na gestão de projetos ou da gestão de projetos sustentáveis, aspectos de ética e legislação, questões sociais, educação e pesquisa, gestão de recursos naturais, desenvolvimento de produtos e processos, e infraestrutura urbana. Porém, é importante ressaltar que muitos artigos ainda referem-se ao termo sustentável considerando apenas o aspecto financeiro, e outras vezes incluem a diretriz ambiental, mas não a diretriz social.

Apesar dos resultados, o estudo apresenta limitações relacionadas à abordagem metodológica utilizada. Primeiramente, a respeito da utilização de uma única base de dados, apesar de sua reconhecida importância no meio acadêmico. A segunda limitação refere-se ao processo de seleção de artigos a serem mantidos na amostra, tanto pela relação simultânea com a sustentabilidade e a gestão de projetos, quanto através do que se considerou de relevância para o tema, assim foram excluídos artigos de alguns temas que em outros pontos de vista poderiam ser considerados mantidos na amostra.

\section{REFERÊECIAS}

AFLAKI, S; KLEINDORFER, P. R.; POLVORINOS, V. S. D. Finding and Implementing Energy Efficiency Projects in Industrial Facilities. Production and Operations Management, v. 22, n. 3, p. 503-517, 2013.

ALMANSI, F; TAMMARAZIO, A. Mobilizing projects in community organizations with a long-term perspective: neighbourhood credit funds in Buenos Aires, Argentina. Environment and Urbanization, v. 20, n. 1, p. 121-147, 2008.

ARASZKIEWICZ, K. Green BIM Concept - Scandinavian Inspirations . Archives of Civil Engineering, v. 62, n. 1, 2016.

BALEZENTIENE, L; STREIMIKIENE, D; BALEZENTIS, T. Fuzzy decision support methodology for sustainable energy crop selection. Renewable and Sustainable Energy Reviews, 17, p.83-93. 2013.

BOCCHINI, P.; FRANGOPOL, D.M.; UMMENHOFER, T.; ZINKE, T. Resilience and sustainability of civil infrastructure: toward a unified approach. Journal of Infrastructure Systems, 20, p.1-16. 2014.

BONOU, A; SKELTON, K; OLSEN, SI Ecodesign framework for developing wind turbines. Journal Of Cleaner Production, v. 126, n. 10, p. 643-653, 2016.

BRONES, F; DE CARVALHO, M.M. From 50 to 1: integrating literature toward a systemic ecodesign model. Journal of Cleaner Production, 96, p.44-57. 2015. 
BRONES, F; DE CARVALHO, M.M.; ZANCUL, E.D. Ecodesign in project management: a missing link for the integration of sustainability in product development?. Journal of Cleaner Production, v. 80, n. 1, p.106-118, 2014.

CARVALHO, M.M.; RABECHINI JR, R. Fundamentos em gestão de projetos: construindo competências para gerenciar projetos. 3a ed. São Paulo: Editora Atlas, 2011.

CAZORLA, A; DE LOS RIOS, I; SALVO, M. Working With People (WWP) in Rural Development Projects: a Proposal from Social Learning. Cuadernos de Desarrollo Rural, v.10, 2013.

COLENBRANDER, D.R.; SOWMAN, M.R. Merging Socioeconomic Imperatives with Geospatial Data: A Non-Negotiable for Coastal Risk Management in South Africa. Coastal Management, v. 43, n. 3, p. 270-300, 2015.

CRAIG, G. A.; SAYDAM, S.; DEMPSTER, A.G. Mining off-Earth minerals: a long-term play?. Journal of The Southern African Institute of Mining and Metallurgy, v. 114, 2014.

CUST, J. Using intermediate indicators: lessons for climate policy. Climate Policy, v.9, 2009.

DE BRUIJNE, M; VAN DE RIET, O; DE HAAN, A; KOPPENJAN, J. Dealing with Dilemma's: How Can Experiments Contribute to a More Sustainable Mobility System?. European Journal of Transport and Infrastructure Research, v. 10, n. 3, 2010.

DE LOS RIOS-CARMENADO, I; ORTUNO, M; RIVERA, M. Private-Public Partnership as a Tool to Promote Entrepreneurship for Sustainable Development: WWP Torrearte Experience. Sustainability. 2016.

ELKINGTON, J. Partnerships from cannibals with forks: The triple bottom line of 21stcentury business. Environmental Quality Management, v.8 (1), p. 37-51, 1998.

FERNÁNDEZ-SÁNCHEZ, G.; RODRÍGUEZ-LÓPEZ, F. A methodology to identify sustainability indicators in construction project management - Application to infrastructure projects in Spain. Ecological Indicators, 10, p.1193-1201. 2010.

GHAPANCHI, A.H. Investigating the Interrelationships among Success Measures of Open Source Software Projects. Journal of Organizational Computing and Electronic Commerce. 2015.

GHOSH, S; et al. Organizational Governance to Integrate Sustainability Projects: A Case Study. Technological and Economic Development of Economy, p. 1-24, 2014.

GÖRG, C. et al. Engaging Local Knowledge in Biodiversity Research: Experiences from Large Inter- and Transdisciplinary Projects. Interdisciplinary Science Reviews, v. 39, p. 323$341,2014$.

GMELIN, H; SEURING, S. Achieving sustainable new product development by integrating product life-cycle management capabilities. International Journal of Production Economics. 2014.

GUERRERO-LIQUET, G.C. et al. Decision-Making for Risk Management in Sustainable Renewable Energy Facilities: A Case Study in the Dominican Republic. Sustainability, 2016.

HART, S.L. MILSTEIN, M.B. Creating sustainable value. Academy of Management Executive, 17(2), pp. 56-67, 2003.

HERAZO, B; LIZARRALDE, G.The influence of green building certifications in collaboration and innovation processes. Construction Management and Economics, 2015. 
HWANG, B.; NG, W.J. Project management knowledge and skills for green construction: Overcoming challenges. International Journal of Project Management, 31, p.272-284. 2013.

HWANG, B. G. ; TAN, J. S. Green building project management: obstacles and solutions for sustainable development. Sustainable Development, v. 20, n. 5, p. 335-349 2012.

ISON, R.; ROLING, N.; WATSON, D. Challenges to science and society in the sustainable management and use of water: investigating the role of social learning. Environmental Science \& Policy, 10, p.499-511. 2007.

JIAO, Y; et al. A cloud approach to unified lifecycle data management in architecture, engineering, construction and facilities management: Integrating BIMs and SNS. Advanced Engineering Informatics, v. 27, n. 2, p. 173-188, 2013.

KAKABADSE, NK; KAKABADSE, AP; SUMMERS, N. Effectiveness of Private Finance Initiatives (PFI): Study of private financing for the provision of capital assets for schools. Public Administration and Development, v. 27, n. 1, p. 49-61, 2007.

LAWS, D; LOEBER, A. Sustainable development and professional practice. Proceedings of The Institution of Civil Engineers - Engineering Sustainability, v.164 (1), p. 25-33, 2011.

LEBE, S.S.; VRECKO, I. Eco-labels and Schemes: A Requisitely Holistic Proof of Tourism's Social Responsibility?. Systems Research and Behavioral Science. 2015.

LEHEIS, S. High-speed train planning in France: Lessons from the Mediterranean TGV-line. Transport Policy, 21, p. 37-44. 2012.

LUIZ, J.V.R. et al. Ecodesign field of research throughout the world: mapping the territory by using an evolutionary lens. Scientometrics, 2016.

MADDEN, P.B.; MORAWSKI, J.D. The Future Of The Canadian Oil Stands: Engineering And Project Management Advances. Energy \& Environment, 2011.

MARCELINO-SADABA, S; GONZALEZ-JAEN, LF; PEREZ-EZCURDIA, A. Using project management as a way to sustainability. From a comprehensive review to a framework definition. Journal of Cleaner Production, v. 99, p. 1-16, 2015.

MARTENS, M. L.; CARVALHO, M. M. The challenge of introducing sustainability into project management function: multiple-case studies. Journal of Cleaner Production, 117, p. 29-40. 2016.

MAYRING, P. Qualitative Content Analysis: Theoretical Foundation, Basic Procedures and Software Solution. Sage, Klagenfurt. 2014.

MIEG, H.A. Sustainability and innovation in urban development: concept and case. Sustainable Development. 2012.

MOLENAAR, K. R.; SOBIN, N.; ANTILLON, E. I. A synthesis of best-value procurement practices for sustainable design-build projects in the public sector. Journal of Green Building, 2010.

OKEIBUNOR, J. et al. A model for evaluating the sustainability of community-directed treatment with ivermectin in the African Program for Onchocerciasis Control. International Journal of Health Planning and Management, v. 27(3), p. 257-271, 2012.

PADE-KHENE, C; MALLINSON, B; SEWRY, D. Sustainable rural ICT project management practice for developing countries: investigating the Dwesa and RUMEP projects. Information Technology For Development. 2011. 
PETTER, S; KHAZANCHI, D; MURPHY, JD. A Design Science Based Evaluation Framework for Patterns. Data Base For Advances In Information Systems. 2010.

PORTER, M.E.; KRAMER, M.R. Creating Shared Value: How to reinvent capitalism - and unleash a wave of innovation and growth. Harvard Business Review, v. 89, p. 62-77, 2011.

ROBICHAUD, L.V.; ANANTATMULA, V.S. Greening Project Management Practices for Sustainable Construction. Journal Of Management In Engineering, v. 27, 2011.

SANCHEZ, M.A. Integrating sustainability issues into project management. Journal of Cleaner Production, v. 96, p. 319-330, 2015.

SANDIN, G. et al. Making the most of LCA in technical inter-organisational R\&D projects. Journal of Cleaner Production. 2014.

SENEVIRATNE, K; AMARATUNGA, D; HAIGH, R. Post conflict housing reconstruction Exploring the challenges of addressing housing needs in Sri Lanka. Built Environment Project, 2015.

SEURING, S.; MÜLLER, M. From a Literature Review to a Conceptual Framework for Sustainable Supply Chain Management. Journal of Cleaner Production, 16(15), p.1699-1710. 2008.

SILVIUS, G.; SCHIPPERB, R.; NEDESKI, S. Sustainability in Project Management: Reality Bites. PM World Journal, v. 2, n. 2, 2013.

TAYLAN et al. Construction projects selection and risk assessment by fuzzy AHP and fuzzy TOPSIS methodologies. Applied Soft Computing, 17, p.105-116. 2014.

TRENCHER et al. University partnerships for co-designing and co-producing urban sustainability. Global Environmental Change-Human And Policy Dimensions, v.28, p.153165, 2014.

VIDAL, M.C. et al. Ergonomic sustainability based on the ergonomic maturity level measurement. Work - A Journal Of Prevention Assessment \& Rehabilitation. 2012.

WCED. Report of the World Commission on Environment and Development: Our Common Future. Geneva: United Nations, 1987.

WEERASINGHE, G; SOUNDARARAJAN, K; RUWANPURA, J. LEED-PDRI framework for pre-project planning of sustainable building projects. Journal of Green Building, 2007.

YANG, R. J.; ZOU, P. X.W. Stakeholder-associated risks and their interactions in complex green building projects: A social network model. Building and Environment, 73, p.208-222. 2014.

YANG, R.J.; SHEN, G.Q.P. Framework for Stakeholder Management in Construction Projects. Journal Of Management In Engineering, v.31(4), 2015.

YORK, J.G.; VENKATARAMAN, S. The entrepreneur-environment nexus: Uncertainty, innovation, and allocation. Journal of Business Venturing, 25 (5), p. 449-463. 2010.

ZAVADSKAS et al. Multiple criteria decision support system for assessment of projects managers in construction. International Journal of Information Technology \& Decision Making, 11(2), p.501-520. 2012.

ZHANG et al. Applying evolutionary prototyping model in developing FIDSS: An intelligent decision support system for fish disease/health management. International Journal of Project Management, 32, p.66-76. 2009. 